\title{
Friction at Nanoscale
}

\section{Chee How Wong*}

School of Mechanical and Aerospace Engineering, Nanyang Technological University, 50 Nanyang Avenue, Singapore 639798, Singapore

The failure to commercialize any silicon-based Micro-ElectroMechanical Systems (MEMS) or Nano-Electro-Mechanical Systems (NEMS) devices that have sliding parts in contact [1] highlights the need for alternative materials for MEMS and NEMS devices. Carbonbased materials are an attractive replacement that could alleviate the problem.

Microsystems, such as MEMS, and nanosystems, such as NEMS, have very little tolerance for friction and wear since the smallest wear particles could result in catastrophic failure. The problem is made worse when liquid lubrication is not a complete solution to reducing friction and wear due to problems associated with stiction, contamination and the formation of meniscus. For instance, typical MEMS components are only a few microns thick. They are structurally unable to withstand high surface traction, which presents a problem since surface forces become increasingly dominant as the physical dimensions become smaller. Yet many of these microsystems, such as magnetic recording systems, are subject to harsh operating conditions which intensify the already challenging task of obtaining low friction solutions.

One possible solution to achieve low friction in microsystems is the use of thin, hard coatings. The hard coatings provide protection against wear and deformation without the use of liquid lubrication. Additionally, the coatings also help to reduce friction, and the coatings typically stay on the substrate well, compared to other surface treatments such as self-assembled monolayers and solid lubricants. Although ceramic is a good candidate for coatings due to their chemical inertness, high temperature tolerance, and good hardness [2-5], amorphous carbon films or more commonly known as Diamond-Like Carbon (DLC) have been extensively studied as a new candidate for coatings due to their excellent mechanical and extremely good tribological performance $[6,7]$. As researchers continue to develop the technology to establish and improve methods for producing DLC coatings with MEMS and NEMS devices, it is necessary to characterize the fundamental surface properties of the DLC coatings.

At macroscopic scale, the mechanical and tribological performances of these carbon-based films are excellent. Diamond, for example, can be grown in nanocrystalline thin film form with mechanical properties comparable to single crystal diamond [8,9]. DLC films, when grown as near-frictionless carbon, exhibits friction coefficients as low as 0.001 and wear rates even lower than diamond [10]. Even though the technology involving DLC films are more mature with widespread use in wear and corrosion protection in many applications [10], the tribological performance of DLC degrades significantly when water is present [11-15].

Furthermore, like many other materials, frictional responses in DLC films exhibit distinct characteristics at different length scales - from macroscopic down to molecular level. Three typical configurational characteristics of frictional sliding resulting from different length scales are: (1) single-asperity contact, (2) multi-asperity contact, and (3) molecular contact.

The frictional response of a single-asperity contact depends predominantly on the size and the shear strength of the adhesive contact junction. The contact size is determined by the elastic response of adhe- sive contact which has been well characterised by various models [1618]. A considerable amount of experiments have also been performed to investigate the single-asperity friction [19-23].

As the length scale gets larger, new approaches are needed to bridge the frictional response from single asperity to multi-asperity contact. In 1957, Archard [24] concluded that the average microcontact state hardly changes whatever the load. Instead only the number of micro-contacts varies, and the multiple micro-contacts would alter the nonlinear frictional response towards a linear response. Later investigations [25-27] attributed the linear response of rough surface friction, as an integrated behaviour of nonlinear singleasperity responses. Macroscopically, friction displayed a complicated dependence on sliding and load histories. These characteristics have been described by the empirical rate and state-variable dependent constitutive formulations [28-30]. However, the underlying micromechanisms are little understood and the physical interpretations of the state variables are still vague.

Despite the myriad of studies, presently, the main obstacle to develop ultra-low friction systems is the lack of fundamental understanding of the mechanisms of friction in the regime of sliding without severe deformation and wear, where friction is determined by the atomic interactions between shearing surfaces. Despite past efforts, a comprehensive understanding of friction at the atomic level is still lacking, and the fundamental behaviour of atoms in the interfacial region has not been characterized well enough to build a useful strategy for the development of low-friction technology.

An obvious strategy for reducing friction is to reduce the strength of interatomic interaction between the surfaces, which can be done by surface treatment with the intention of reducing the free surface energies, for example, or by deliberately choosing weakly adhering materials. This is not unlike the macroscopic strategy of lowering friction by selecting metallurgically incompatible materials. However, there is a logical inconsistency in the belief that low adhesion leads to low friction, since the former is related to the resistance against separation of the surfaces while the latter is related to the resistance against their relative translation. In addition, there is actually no standard that relates adhesion and friction, which means that it is not known how much reduction in friction can be expected per unit reduction of adhesion.

It is in this regard that the study of the fundamental characteristics of friction and wear of DLC films at nano-scale level is important. Studies in this area are important not just for nanotechnology applications, but for fundamental science as well. Although many practical, macroscopic

*Corresponding author: Chee How Wong, School of Mechanical and Aerospace Engineering, Nanyang Technological University, 50 Nanyang Avenue, Singapore 639798, Singapore, E-mail: chwong@ntu.edu.sg

Received July 26, 2012; Accepted July 28, 2012; Published July 31, 2012

Citation: Wong CH (2012) Friction at Nanoscale. J Appl Mech Eng 1:e103. doi:10.4172/ 2168-9873.1000e103

Copyright: ( 2012 Wong $\mathrm{CH}$. This is an open-access article distributed under the terms of the Creative Commons Attribution License, which permits unrestricted use, distribution, and reproduction in any medium, provided the original author and source are credited. 
engineering applications involving tribology have been successfully addressed, relatively little is understood about the underlying fundamental mechanisms of friction at nano-scale. As a result, there are no commercially viable MEMS and NEMS devices that involve contacting and sliding interfaces, despite ample capabilities to fabricate such devices. These effects are even more drastic at the nano-scale level, where newly discovered paradigms govern interactions during contact and relative motion $[21,31,32]$. Therefore, the study of friction at nanoscale may be the key to uncovering the true interactions between two complex, multi-asperity surfaces at larger scales.

\section{References}

1. Maboudian R, Ashurst WR, Carraro C (2002) Tribological Challenges in Micromechanical Systems. Tribology Letters 12: 95-100.

2. Jamal T, Nimmagadda R, Bunshah RF (1980) Friction and adhesive wear of titanium carbide and titanium nitride overlay coatings. Thin Solid Films 73: 245254

3. Wiklund U, Wänstrand O, Larsson M, Hogmark S (1999) Evaluation of new multilayered physical vapour deposition coatings in sliding contact. Wear 236: 88-95.

4. Bienk EJ, Reitz H, Mikkelsen NJ (1995) Wear and friction properties of hard PVD coatings. Surface and Coatings Technology 77: 475-480.

5. Hogmark S, Jacobson S, and Larsson M (2000) Design and evaluation of tribological coatings. Wear 246: 20-33.

6. McKenzie DR (1996) Tetrahedral bonding in amorphous carbon. Reports on Progress in Physics 59: 1611

7. Robertson J (1986) Amorphous Carbon. Advances in Physics 35: 317-374.

8. Auciello O, Gruen DM, Krauss AR, Jayatissa A, Sumant A, et al. (2001) Science and technology of ultrananocrystalline diamond (UNCD) thin films for multifunctional devices. Smart Structures and Devices,USA

9. Philip J, Hess P, Feygelson T, Butler JE, Chattopadhyay S, et al. (2003) Elastic mechanical, and thermal properties of nanocrystalline diamond films. Journa of Applied Physics 93: 2164-2171.

10. Erdemir A, Donnet C (2001) Tribology of Diamond, Diamond-like Carbon and Related Films. Modern Tribology Handbook, CRC Pres, Florida, USA

11. Andersson J, Erck RA, Erdemir A (2003) Frictional behavior of diamondlike carbon films in vacuum and under varying water vapor pressure. Surface and Coatings Technology 164: 535-540.

12. Dickrell PL, Sawyer WG, Heimberg JA, Singer IL, Wahl KJ, et al. (2005) A GasSurface Interaction Model for Spatial and Time-Dependent Friction Coefficient in Reciprocating Contacts: Applications to Near-Frictionless Carbon. Journal of Tribology 127: 82-88.

13. Heimberg JA, Wahl KJ, Singer IL, Erdemir A (2001) Superlow friction behavior of diamond-like carbon coatings: Time and speed effects. Applied Physics Letters 78: 2449-2451.

14. Gao F, Erdemir A, Tysoe W (2005) The Tribological Properties of Low-friction Hydrogenated Diamond-like Carbon Measured in Ultrahigh Vacuum. Tribology Letters 20: 221-227.

15. Kim HI, Lince JR, Eryilmaz OL, Erdemir A (2006) Environmental effects on the friction of hydrogenated DLC films. Tribology Letters 21: 51-56.

16. Johnson KL, Kendall K, Roberts AD (1971) Surface Energy and the Contact of Elastic Solids. Proceedings of the Royal Society of London. Series A, Mathematical and Physical Sciences 324: 301-313.

17. Derjaguin BV, Muller VM, Toporov YP (1975) Effect of contact deformations on the adhesion of particles. J Colloid Interface Sci 53: 314-326.

18. Maugis D (1992) Adhesion of spheres: The JKR-DMT transition using a dugdale model. J Colloid Interface Sci 150: 243-269.

19. Carpick RW, Agrait N, Ogletree DF, Salmeron M (1996) Variation of the Interfacial Shear Strength and Adhesion of a Nanometer-Sized Contact. Langmuir 12: 3334-3340.

20. Lantz MA, O'Shea SJ, Welland ME, Johnson KL (1997) Atomic-force- microscope study of contact area and friction on NbSe2. Phys Rev 55: 10776 . 10785.

21. Carpick RW, Salmeron M (1997) Scratching the Surface: Fundamenta Investigations of Tribology with Atomic Force Microscopy. Chem Rev 97: 11631194.

22. Dedkov GV (2000) Experimental and Theoretical Aspects of the Modern Nanotribology. Physica Status Solidi( a) 179: 3-75.

23. Gnecco E, Bennewitz R, Gyalog T, Meyer E (2001) Friction experiments on the nanometre scale. J Phys: Condens Matter 13: R619.

24. Archard JF (1957) Elastic Deformation and the Laws of Friction. Proceedings of the Royal Society. A, Mathematical, Physical, and Engineering Sciences 243 190-205.

25. Greenwood JA, Williamson JBP (1966) Contact of Nominally Flat Surface. Proceedings of the Royal Society. A, Mathematical, Physical, and Engineering Sciences 295: 300-319.

26. Greenwood JA, Tripp JH (1967) The Elastic Constant of Rough Spheres Journal of Applied Mechanics 34: 153-159.

27. Greenwood JA, Tripp JH (1970) The Contact of Two Nominally Flat Rough Surface. Proceedings of the Institution of Mechanical Engineers 185: 625-633.

28. Dieterich JH (1978) Time-dependent Friction and the Mechanics of Stick-slip. Pure and Applied Geophysics 116: 790-806.

29. Rice JR, Ruina AL (1983) Stability of Steady Frictional Slipping. Journal of Applied Mechanics 50: 343-349.

30. Ruina A (1983) Slip Instability and State Variable Friction Laws. Journal of Geophysical Research 88: 10359-10370.

31. Singer IL (1992) Solid Lubrication Processes. Fundamentals of Friction: Macroscopic and Microscopic Processes, Springer.

32. Bhushan B (1999) Handbook of Micro/Nano Tribology. Boca Raton: CRC Press. 